\title{
Réception des communications et cognition non consciente : les effets non-verbalisables de la communication commerciale
}

Didier Courbet

\section{(2) OpenEdition \\ 12 Journals}

Édition électronique

URL : https://journals.openedition.org/communicationorganisation/2425

DOI : 10.4000/communicationorganisation.2425

ISSN : $1775-3546$

Éditeur

Presses universitaires de Bordeaux

Édition imprimée

Date de publication : 1 novembre 2000

ISSN : 1168-5549

Référence électronique

Didier Courbet, «Réception des communications et cognition non consciente : les effets nonverbalisables de la communication commerciale ", Communication et organisation [En ligne], 18 | 2000, mis en ligne le 27 mars 2012, consulté le 05 août 2021. URL : http://journals.openedition.org/ communicationorganisation/2425; DOI : https://doi.org/10.4000/communicationorganisation.2425

Ce document a été généré automatiquement le 5 août 2021.

(c) Presses universitaires de Bordeaux 


\title{
Réception des communications et cognition non consciente : les effets non-verbalisables de la communication commerciale
}

\author{
Didier Courbet
}

Dans le domaine de la réception des communications, de récentes évolutions nous incitent à (ré)orienter les recherches vers les processus non conscients. En suivant Cheesman et Merikle (1986), la conscience est définie comme « la capacité pour un sujet à produire intentionnellement une réponse symbolique (e.g. verbale) à propos d'informations à discriminer (e.g. processus intrapsychique ou événement de l'environnement)». Dit plus simplement, est non conscient ce qui ne peut pas être verbalisé. Dans les études sur la réception non consciente, on ne va utiliser le langage verbal qu'en tant qu'outil de mise en évidence indirecte des processus non conscients. Des évolutions théoriques, méthodologiques et épistémologiques nous conduisent à (ré)ouvrir un tel programme de recherches en sciences de la communication.

\section{Les nouvelles questions générées par l'articulation cognition-communication}

2 Les études de réception sont inséparables d'une conception théorique de la communication, conception qui va orienter le choix des disciplines mobilisées pour l'étude des phénomènes de communication. L'objectif est ici d'intégrer les recherches dans le domaine de la cognition et, plus particulièrement, de la cognition non consciente dans le champ des sciences de la communication. En effet, l'arrivée du paradigme cognitif dans les sciences humaines et sociales et, plus précisément, en psychologie et psychologie sociale, a permis l'apparition de nouveaux modèles et concepts tels l'apprentissage et la mémoire implicites, les traitements automatiques de l'information (traitements non-verbalisables et très rapides) qui se mettent notamment 
en route en parallèle des traitements contrôlés (verbalisables et conscients). Fonctionnant au sein d'un système d'échange socio-économique à multiples enjeux, la communication des marques est fondamentalement un processus finalisé dans lequel des acteurs socio-économiques, dotés d'intentionnalité et cherchant à réaliser des objectifs, fabriquent un dispositif pour influencer des acteurs psycho-socioéconomiques, dotés également d'intentionnalité. En suivant le principe de la pragmatique de la communication, ce n'est qu'en allant étudier les acteurs et leurs représentations dans leur contexte d'évolution habituel que peuvent être étudiés scientifiquement la communication et son fonctionnement. On continue cependant à voir, ça et là, des études sémiologiques ou sémiotiques, reposant donc sur l'ancien modèle du code, qui empiètent sur le terrain de la réception. Le récepteur fait bien autre chose que produire des significations comme le décrivent ces disciplines. De plus, celles-ci ne disent rien sur le processus psychosocial de construction des significations. Grâce aux études de réception, nous tentons également de montrer les limites des théories de la communication reposant soit sur le principe de « co construction d'une réalité » soit sur le principe d'inférence des intentions à partir d'indices (pragmatique linguistique).

3 En dépassant le titre volontairement accrocheur de cet article, il convient bien entendu de faire évoluer l'ancienne dichotomie simpliste " récepteur actif vs récepteur passif ». L'objectif est de mieux connaître les processus cognitifs et les traitements de l'information par lesquels les personnes forment ou changent leurs opinions ou leurs représentations suite aux communications des marques ainsi que les processus qui transforment ces effets attitudinaux en réelles actions. Nous ne parlerons ici que des traitements non conscients (pour une théorie plus générale de la réception, voir Courbet, 1999). Après avoir répondu par l'affirmative aux questions : " existe-t-il des influences et des effets non conscients des communications des marques?", "ces effets non conscients sont-ils différents des effets conscients?» (les réponses sont d'ailleurs bien plus complexes que ce qui est théorisé dans les célèbres « conditionnement classique » ou « seringue hypodermique », voir Fourquet. 1999). les recherches actuelles tentent d'expliquer les processus sériels, parallèles et en cascade qui sous-tendent ces effets :

Comment s'articulent les trois types de traitements non conscients (structuraux, sémantiques et affectifs) pendant la réception? Quels sont les liens entre les traitements inconscients qui se déroulent en parallèle de tous traitements conscients des messages, les pensées et les conduites?

\section{Évolutions méthodologiques et épistémologie transversale}

5 Sur le plan épistémologique, la transversalité des approches françaises de la communication permet d'associer des démarches issues des paradigmes herméneutiques et constructivistes avec des démarches auparavant revendiquées par le seul paradigme positiviste. En adhérant au principe de vérification et de preuve que l'on retrouve dans ces deux types d'épistémologies, il est possible d'articuler, d'une part, des modélisations locales qui circonscrivent un événement précis, bien délimité et composé de quelques facteurs parfaitement définis un à un avec, d'autre part, des 
théories plus générales de la communication qui représentent une multitude d'événements en interdépendance et contextualisés.

6 S'insérant dans cette épistémologie transversale, les méthodes que nous utilisons dans ces recherches allient à la fois l'observation naturaliste, l'entretien et l'expérimentation. Dans ses grandes lignes, la démarche consiste, tout d'abord, à observer les récepteurs de messages de communication externe des organisations (e.g. la publicité) dans la vie quotidienne puis à les interroger pour émettre des hypothèses sur les modes d'influence et sur les effets. Il s'agit ensuite, dans la phase expérimentale, de diviser aléatoirement un grand groupe de personnes en deux petits groupes et d'exposer les personnes de chaque groupe, au cours d'un moment de leur vie quotidienne, à un nouveau message publicitaire (sans qu'ils sachent qu'une quelconque étude est menée). Un seul facteur varie entre les expositions des deux groupes : c'est le facteur sur lequel une hypothèse a été émise. On pose ensuite les mêmes questions aux personnes des deux groupes. Comme les conditions sont identiques par ailleurs, la variation des réponses d'un groupe à l'autre est due au facteur qu'on a fait varier. Si cette différence va dans le sens de nos hypothèses, celles-ci ont des chances d'être valides dans cette situation là (ou, plus exactement, de ne pas être fausses). Après avoir limité la validité des résultats aux circonstances de la vie quotidienne qui correspondent à la situation observée, on les replace, par un mouvement d'induction, dans une théorie plus générale de la communication que l'on va ainsi progressivement enrichir. Fidèle à cette démarche de vérification, nous reléguons les théories psychanalytiques sur les influences non conscientes (et donc «non-verbalisables») de la publicité au rang d'uniques corpus d'hypothèses qu'il est encore nécessaire, quand c'est possible, d'opérationnaliser et de tester.

7 Dans les études de réception, si les chercheurs n'utilisent pas des méthodes pertinentes, aucun traitement non conscient ne pourra être mis en évidence et $a$ fortiori étudié. Avec les méthodes classiquement utilisées dans le "paradigme du récepteur actif (e.g. simples entretiens ou questionnaires passés auprès des récepteurs; observations systématisées des comportements; analyses de contenu de messages; introspection de l'analyste), on ne peut que conclure à l'absence d'influence non consciente des communications. Étroitement dépendantes des progrès technologiques, les techniques expérimentales utilisées dans les études sur les influences non conscientes peuvent être classées en deux catégories :

8 les techniques de présentation des messages : soit par des médias classiques, soit par tachytoscope ou ordinateur qui permettent d'exposer les individus à certaines images présentées tellement rapidement (quelques millisecondes) qu'elles ne peuvent être détectées consciemment ;

les techniques d'enregistrement des réponses verbales ou non-verbales des récepteurs : on peut observer des effets en dehors de toute conscience du sujet soit dans le contenu du langage (grâce à une analyse indirecte : tests indirects; tests projectifs), soit dans le temps que le sujet met à répondre (enregistré par ordinateur). Certaines recherches utilisent des indices physiologiques, la technique des potentiels évoqués enregistrés par électroencéphalogramme et l'imagerie par résonance magnétique nucléaire.

Pour illustrer, prenons le cas banal d'une personne qui fait ses courses dans un hypermarché. On peut maintenant expliquer pourquoi, lorsqu'elle est faiblement impliquée par un produit, elle oriente son choix, sans même en avoir conscience, sur une marque qui a fait de la publicité. Souvent la personne est elle-même incapable 
d'expliquer pourquoi et ne se souvient absolument pas avoir été exposée à la publicité. On peut même montrer que les éventuelles raisons qu'elle peut donner pour justifier son choix ne sont pas les véritables raisons. Comment la communication de cette marque peut, sans que la personne en soit consciente, engendrer une telle attitude favorable et même l'achat du produit? Nous expliquons quatre modes d'influence non consciente.

\section{Les traitements automatiques des messages perçus sans conscience}

11 Ce type de processus non conscients s'observe par exemple chez une personne qui, située à proximité d'un poste de radio ou de télévision allumé, focalise son attention sur une toute autre tache. De nombreux supports expérimentaux nous confirment que la publicité d'une marque peut être traitée sans conscience par cette personne. Non seulement le psychisme traite les représentations linguistiques de cette marque (son nom, e.g. "Skip») et les reconnaît si elles sont déjà stockées en mémoire, mais des traitements sémantiques ont également lieu sans conscience. En effet, pendant un temps très court (de l'ordre de la seconde) certaines représentations sémantiques associées dans le réseau mémoriel au secteur de la lessive et du linge sont alors activées. Si cette même expérience se reproduit plusieurs fois, ces traitements automatiques vont provoquer une plus grande accessibilité des représentations de la marque en mémoire. C'est alors sur le point de vente que la deuxième étape de l'influence va s'opérer. Lorsque le consommateur voit la marque dans un hypermarché, sa vision va immédiatement provoquer une très vague impression de familiarité. Incapable d'expliquer les raisons de cette impression, le psychisme va alors automatiquement et immédiatement juger la marque en attribuant faussement cette familiarité : par cette "mésattribution », la marque sera alors jugée favorablement. C'est en partant des travaux en psychologie cognitive de Jacoby (e.g. Jacoby et Whitehouse, 1989) sur la fluidité perceptive (perceptual fluency) puis ceux de Bornstein au début des années 90 (voir Bornstein et Pittman, 1992) qu'en France, Channouf (voir ci-dessous) dans le domaine des images subliminales et Courbet en communication des entreprises ont mis en place un programme de recherches pour mieux comprendre ce processus de mésattribution de la familiarité.

\section{Les traitements parallèles de procédés perçus sans conscience}

La communication des marques, notamment à la télévision, est ainsi faite que lorsque le téléspectateur porte attention à certains éléments du message, d'autres éléments sont traités au même moment de manière non consciente.

Ces derniers interfèrent avec les traitements conscients (Courbet, 2000a). C'est notamment le cas des procédés intentionnellement fabriqués pour faire générer, de manière subreptice des émotions positives en parallèle des traitements conscients $d u$ contenu argumentaire (e.g. musique agréable en publicité ; apparition de la marque au sein d'un programme qui déclenche des émotions positives). Le processus de réception d'une personne dans un état affectif positif est alors tout à fait particulier. Il juge le 
message non pas en fonction d'une analyse détaillée des arguments ou du contenu mais de manière beaucoup plus globale à partir d'un sentiment général. Les émotions positives consomment une partie des ressources de la mémoire de travail et conduisent ainsi à évaluer rapidement le message avec des règles simples de décision (appelées heuristiques, voir par exemple Forgas, 1994). Plus simplement, l'individu est alors incapable de traiter de manière approfondie le message publicitaire. Les conclusions tirées par le récepteur sont alors d'autant plus favorables au contenu que celui-ci montre une scène séduisante ou chargée affectivement. En effet dans de nombreux messages, les publicitaires associent la marque à plusieurs stimulus qui déclenchent des réactions affectives positives mais qui n'ont aucun lien fonctionnel avec le produit (une personne nue. une vedette du show-business, un bébé...). Un traitement conscient et approfondi d'un tel message conduirait logiquement, d'une part, à émettre envers cette association artificielle des réponses cognitives de contre argumentation et, d'autre part, de rejeter le contenu. Or l'affect positif déclenché par ces stimulus ainsi que d'autres procédés publicitaires (images rapides, humour, effet de surprise...) permettent, d'une part, de ne pas éveiller la conscience des sujets et, d'autre part, d'accepter au sein d'un même spot des rapprochements aussi artificiels qu'une bouteille de gaz avec un joli chien, qu'un savon avec une vedette de cinéma ou d'un restaurant rapide avec la joie d'un nouveau-né. Ici la marque et l'élément affectivement investi sont vus consciemment: c'est sur l'artificialité de leur association que porte l'absence de conscience.

Ce principe est également utilisé dans la technique du parrainage télévisuel. Présent sur l'ensemble des chaînes françaises depuis 1987, ce moyen de communication opère de manière extrêmement furtive. Si une marque apparaît pendant ou juste avant une émission, les téléspectateurs devraient s'en étonner à cause justement de l'absence d'un quelconque lien logique entre la marque et le programme. Or les courtes séquences de présentation du parrain sont conçues pour ne pas éveiller l'attention et ne pas provoquer un tel rejet (appelé dans ce cas effet de réactance) : tant au niveau formel que sémantique, la séquence semble s'intégrer naturellement à l'émission (Courbet, 1999, p. 131-199). L'influence opère alors sans conscience. La présence de la même marque depuis des années à proximité de la météo conduit les téléspectateurs à l'inclure dans des "scripts» mémoriels: la marque fait partie du quotidien de l'individu au même titre que la météo elle-même. Lors de chaque nouvelle vision, les défenses psychologiques des téléspectateurs sont inexistantes. la répétition peut alors tranquillement faire son effet. Cette influence non consciente est ici d'autant plus forte que les programmes sont investis affectivement et souvent attendus avec impatience. Cet exemple montre qu'on a affaire à des mécanismes d'influence non conscients même quand l'individu voit consciemment le message : il ignore cependant quels éléments agissent sur lui et comment ils agissent.

\section{Les effets des messages perçus consciemment mais oubliés}

15 À l'aide de la méthode expérimentale, nous avons montré (Courbet, 2000b) que des personnes peuvent aimer une toute nouvelle marque et penser que ses produits sont luxueux (haut de gamme) sans savoir pourquoi ni se souvenir de l'avoir déjà vue auparavant. Or ces personnes ont oublié qu'elles ont bel et bien été exposées, une 
semaine auparavant, à deux messages très courts ( 6 secondes) de cette nouvelle marque juste avant et après une émission gaie. Un deuxième groupe de personnes avaient vu cette nouvelle marque, également pour la première fois, dans les mêmes conditions et pendant la même durée juste avant et après un programme triste. Une semaine après, ces derniers individus, toujours sans se souvenir de l'avoir déjà vu et sans savoir pourquoi, l'ont comparativement moins apprécié et ont jugé ces produits comme étant moins luxueux (davantage bas de gamme). C'est donc la seule association entre une marque et des programmes déclenchant des émotions différentes qui a amené à évaluer différemment la marque, et ce, en dehors de toute conscience des processus d'influence. L'introspection ne permet donc pas d'accéder à ce types de représentations mnésiques car celles-ci ne peuvent être verbalisées. Or elles agissent sur les jugements et les comportements : elles sont stockées en mémoire dite implicite. Les sujets ont ainsi faussement l'impression qu'ils sont autonomes dans leur décision, pourtant ils ont élaboré un jugement sans connaître les véritables déterminants de leur raisonnement. Dans ce cas, la persuasion n'est efficace que si le processus est non conscient et le message est réellement oublié. En effet, si le sujet se souvient du message ou s'il prend conscience qu'on cherche à l'influencer à son insu, il procède de lui-même à des corrections inhibant ainsi la persuasion : la connaissance des techniques publicitaires diminue donc leur efficacité (voir Greenwald et Banaji, 1995).

\section{Les effets de la publicité subliminale}

Nous appelons stimulus subliminaux des stimulus enregistrés par le système sensoriel d'une personne mais qui ne peuvent être détectés consciemment même si l'individu focalise son attention (c'est le cas de la fameuse «25e image $»^{1}$. Interdite en France par l'article 10 du décret sur l'audiovisuel du 27 mars 1992, la publicité subliminale a fait l'objet de multiples travaux scientifiques. Au contraire de ce qu'ont pu en dire les rumeurs puis les contre rumeurs, les recherches empiriques en psychologie, en psychologie sociale et en neurosciences menées depuis 15 ans sont formelles : employés dans certaines conditions, les stimulus subliminaux ont des effets indéniables (voir Channouf et Pichevin, 1998 ; Channouf, 2000). Aussi s'agit-il plus précisément de mieux connaître la nature de ces effets, leur puissance, leur stabilité temporelle, les processus psychologiques qui les sous-tendent, le type de stimulus qui les provoquent mais également leurs limites. En fait, les effets de la publicité subliminale ne s'observent que dans certaines conditions précises, conditions que de nombreuses recherches n'ont pas mises en place. Ces dernières ont ainsi conclu, un peu rapidement, à son inefficacité (par exemple la méta-analyse de ïrappey, 1996).

Il faut, tout d'abord, que le message soit simple c'est-à-dire constitué d'une seule image ou de quelques mots. La durée de présentation est comprise entre $1 \mathrm{~ms}$ et $50 \mathrm{~ms}^{2}$. Les effets s'observent principalement dans deux cas :

1) Lorsque la présentation est répétée (entre 3 et 15 expositions environ). Si on montre ensuite au sujet plusieurs images parmi lesquelles l'image en question en lui demandant s'il reconnaît celle à laquelle il a été exposé (test direct), sa réponse est négative. Cependant quand on lui demande quelle image il préfère parmi celles qu'on lui présente, il choisit plus souvent l'image à laquelle il a été exposé de manière subliminale (expérience de Moreland et Zajonc; voir Courbet, 1999 pour une discussion). Il est en outre étonnant de voir que ces effets croissent lorsqu'on augmente 
le délai entre l'exposition et la phase de choix : une semaine après les effets sont encore plus puissants (Bornstein, 1989). Dans ce cas également, l'explication la plus plausible est celle de la mésattribution de la familiarité. $\mathrm{Si}$ on recommence cette même expérience soit en montrant ouvertement au sujet, avant qu'il ne fasse son choix, à quelle image il a été exposé, soit avec une image non subliminale, l'effet disparait. La personne semble mettre en place une stratégie de défense en inhibant les effets non conscients (Kihlstrom, 1987). L'impact du subliminal est donc plus fort que l'impact des images vues consciemment. Il semblerait logique que ces effets soient essentiellement observables dans le cas de publicité pour des produits de faible implication pour lesquels la décision d'achat est prise très rapidement dans le magasin.

2) Lorsque le contenu du message flatte une motivation existante de manière latente chez le récepteur. Appliquant de façon subtile à la publicité l'hypothèse de Silverman (1983), Channouf et coll. (1999) ont montré que lorsque des individus sont exposés à des images subliminales représentant une bouteille d'une marque donnée ou un verre d'eau pétillante, ils ont tendance à accepter plus facilement de boire une boisson qu'on leur propose immédiatement après. L'individu ne se rend compte que sa soif est éveillée qu'au moment où il aperçoit la boisson. S'il a le choix, il va préférer la boisson qu'il a l'habitude de consommer (mais non la marque qui figure sur l'image subliminale). Par exemple, si les spectateurs dans un cinéma sont exposés à une publicité subliminale pour Coca-Cola et si on leur propose juste après plusieurs marques, ils vont choisir leur marque habituelle, mais pas forcément Coca-Cola.

\section{Conclusion : deux implications pour les SIC}

20 À condition de les contextualiser au sein d'une épistémologie transversale et de les insérer dans une théorie plus générale de la communication, les études de réception sont d'un apport indéniable pour les sciences de l'information et de la communication. Loin de souscrire à un positivisme acharné, on peut cependant affirmer que, grâce à la méthode expérimentale.

on commence à mieux connaitre de nombreux processus non conscients intervenant dans la réception. Ils étaient jusqu'à présent inaccessibles par les méthodes d'enregistrement des verbalisations, employées seules et en dehors d'un protocole de recherche systématisé. En élargissant le domaine de la communication des organisations et des communications persuasives à d'autres phénomènes de communication, nous pourrons sans doute montrer que bien des explications et des justifications que les acteurs d'un système communicationnel donnent à leurs propres pensées, inférences, intentions ou actes, ainsi que celles qu'ils attribuent aux autres acteurs, n'ont rien à voir avec les véritables déterminants qui, quant à eux, ne sont pas verbalisables. Dans les cas étudiés, les intentions des producteurs peuvent être effectivement réalisées (et donc la communication parfaitement fonctionner) : - sans qu'il y ait production de significations en réception. Il convient dès lors de compléter les conceptions de la communication fondées sur l'unique logique de la signification (e.g sémiologie, sémiotique, sémantique...).

23 - sans qu'il y ait interprétation d'indices ou inférence des intentions communicatives et informatives en réception. Il convient dès lors de compléter les théories de la communication fondées uniquement sur une logique de «compréhension 
intersubjective" (théories linguistiques ou pragmatiques classiques : e.g. théorie de Sperber et Wilson, 1986).

\section{BIBLIOGRAPHIE}

BORNSTEIN R.F., « Exposure and affect : Overview and Meta-analysis of Research, 1968-1987 », Psychological Bulletin, 106, 265-289, 1989. c R.F. et PITTMAN T.S (Eds.), Perception without Awareness, The Guilford Press, New York, 1992.

CHANNOUF A. et PICHEVIN M.F. (dir.), Le pouvoir subliminal. Delachaux et Niestlé, Paris, 1998.

CHANNOUF A., CANAC D., GOSSET O., «Les effets non spécifiques de la publicité subliminale ». Revue Européenne de Psychologie Appliquée, vol. 49, 1, p. 13-19, 1999.

CHANNOUF A., Les images subliminales. Presses Universitaires de France (Coll. Médecine et Société, Paris, 2000.

CHEESMAN J. et MERIKLE P.M.. « Distinguishing conscious from unconscious perceptual processes ", Canadian Journal of Psychology, 40, 343-367, 1986. COURBET D., Puissance de la Télévision, Stratégies de communication et influence des marques, L'Harmattan (Coll. Communication). Paris. 1999. Courbet D., «L'influence de la publicité et de la communication des marques à la télévision : nouvelles voies de recherche », Humanisme et Entreprise, 239, pp. 1-12. (2000a).

COURBET D., « Les effets automatiques du parrainage télévisuel sur la marque : étude de la mésattribution de la familiarité, du transfert sémantique et de l'influence des émotions déclenchées par le programme ». Recherche et Applications en Marketing (Editions PUG et CNRS), vol. 15, 1. 30 p., (2000b)

FORGAS J.-P., « The Rôle of Emotion in Social Judgements : an Introductory Rcview and an Affect Infusion Model (AIM) », European Journal of Social Psychology, 24, pp. 1-24, 1994.

FOURQUET M.P., « Un siècle de théories de l'influence : histoire du procès des médias », MEI Médiation et Information, $\mathrm{n}^{\circ} 10$ (Histoire et Communication, sous la direction de P. Lardellier), pp. 101-116, 1999.

GRENNWALD A.G. et BANAJI M.R., " Implicit social cognition : attitudes, self esteem and sterreotypes », Psychological Review, 102,1 pp. 4-27, 1995. Traduction française : « La cognition sociale implicite » in Channouf A. et Pichevin M.F. (dir.). Le pouvoir subliminal, Delachaux et Niestlé, pp. 225-297, 1998.

JACOBY L.L. et WHITEHOUSE K., « An illusion of memory : False récognition influenced by unconscious perception ». Journal of Experimental Psychology : General, 118, pp. 126-135, 1989.

KIHLSTROM J.-F.,. « The cognitive unconscious ». Science, 237, pp. 1145-1152, 1987.

SILVERMAN L.H., " The subliminal psychodynamic method : Overview and comprehensive listing of studies », In MASLING (Ed.), Empirical studies of psychoanalytic theory. vol. 1, Hillsdale. NJ : Erlbaum, pp. 69-104, 1983. SPERBER D. et WILSON D.. Relevance. Communication and Cognition, 1986. (trad. française ; La pertinence. Communication et cognition. Editions de Minuit, Pans, 1989). 
TRAPPEY C, «A meta-anlysis of consumer choice and subliminal advertising », Psychology and Marketing, 13. 5, pp. 517-530, 1996

\section{NOTES}

1. Nous ne parlerons ni des «stimulus masqués » comme une image phallique dans une publicité (qui peut se détecter consciemment si on analyse en détail le message), ni des «messages en filigrane « comme les enregistrements à l'envers de paroles sataniques dans certaines chansons. Leurs effets seraient nuls.

2. Pour cette limite supérieure, il faut prendre quelques précautions méthodologiques, voir Channouf (2000).

\section{AUTEUR}

DIDIER COURBET

Université de Nice-Sophia Antipolis 